Article

\title{
A Vacuum of Quantum Gravity That is Ether
}

\author{
Sergey Cherkas 1,+®0000-0002-6132-3052 and Vladimir Kalashnikov 2,+®0000-0002-3435-2333 \\ 1 Institute for Nuclear Problems, Bobruiskaya 11, Minsk 220030, Belarus; cherkas@inp.bsu.by \\ 2 Dipartimento di Ingegneria dell'Informazione, Elettronica e Telecomunicazioni, Sapienza Universitá di \\ Roma, Via Eudossiana 18, 00189 - Roma, RM, Italia; vladimir.kalashnikov@uniroma1.it \\ * Correspondence: vladimir.kalashnikov@uniroma1.it \\ + These authors contributed equally to this work.
}

\begin{abstract}
The fact that quantum gravity does not admit a co-variant vacuum state has far-reaching consequences for all physics. It points out that space could not be empty, and we return to the notion of an ether. Such a concept requires a preferred reference frame for, e.g., universe expansion and black holes. Here, we intend to discuss vacuum and quantum gravity from three essential viewpoints: universe expansion, black holes existence, and quantum decoherence.
\end{abstract}

Keywords: vacuum energy; preferred reference frame; vacuum state; quantum gravity

\section{Introduction.}

From the earliest times, people are thinking how empty is a "nothing," i.e., a space-time, which contains no matter. Some believe that a "nothing" is unreachable, and space-time always contains "something," i.e., ether [1]. If ether is like some matter, then there must be a reference frame at which ether is at rest "in tote," i.e., it represents some stationary medium. After the development of the quantum field theory (QFT), it was founded that the vacuum actually contains a number of virtual particles-antiparticles pair which are created from vacuum and then annihilated during the time $\Delta t=\frac{1}{m}$, where $m$ is a particle mass. That leads to the experimentally observable effects such as anomalous electron magnetic moment, Lamb shift of atomic levels [2] and proves that a vacuum is not empty. However, a soup of the virtual particles-antiparticles pairs is not ether and does not prevent freely motion of the test particle, because QFT vacuum state is invariant relatively to the Lorentz transformations of coordinates.

Taking a gravity into account seems insists more on the existence of ether and preferred reference frame mainly due to difficulties with gravity quantization. In particular Einstein-Aether [3] and Horava-Lifshitz [4] theories have been developed (see [5,6] for phenomenological implications).

Another argument for the existence of the proffered system of reference is the vacuum energy problem. If the zero-point energy is real, one needs to explain why it does not influence universe expansion. One of the solutions is modifying the gravity theory. That may violate invariance relatively to the general transformation of coordinates, e.g., as it is in the Five Vectors Theory (FVT) of gravity, where local Lorentz symmetry is also violated [7]. It should also mention CPT invariance violation [8], which have a number of manifestations both under the Minkowski space-time $[9,10]$ and in the presence of gravity $[6,11]$.

\section{Vacuum state and QG}

Without knowing the quantum gravity (QG) theory, one could hardly say definitely about a vacuum state. However, even toy QG models could provide some suggestions regarding this issue. In particular, one could try to build a model in which gravity is represented only by a spatially nonuniform 
scale factor $a(\eta, r)$. Certainly, within the GR frameworks, such a model is not self-consistent [12]. Nevertheless, there exists the (1+1)- dimensional toy model [12] including a scalar field $\phi(\tau, \sigma)$ and a scale factor $a(\tau, \sigma)$ :

$$
S=\int L d \tau=\frac{1}{2} \int\left(-a^{\prime 2}+\left(\partial_{\sigma} a\right)^{2}+a^{2}\left(\phi^{\prime 2}-\left(\partial_{\sigma} \phi\right)^{2}\right)\right) d \sigma d \tau,
$$

where $\tau$ is a time variable, $\sigma$ is a spatial variable, and prime denotes differentiation over $\tau$. Here, as in GR, the fields evolve on the curved background $a(\tau, \sigma)$, which is, in turn, determined by the fields (the only scalar field $\phi(\tau, \sigma)$ is considered, but there could be a lot of them). The relevant Hamiltonian and momentum constraints, written in the terms of momentums $\pi(\sigma) \equiv \frac{\delta L}{\delta \phi^{\prime}(\sigma)}=a^{2} \phi^{\prime}$, $p_{a}(\sigma) \equiv-\frac{\delta L}{\delta a^{\prime}(\sigma)}=a^{\prime}$

$$
\begin{array}{r}
\left.\mathcal{H}=\frac{1}{2}\left(-p_{a}^{2}-\left(\partial_{\sigma} a\right)^{2}+a^{2}\left(\pi^{2}+\partial_{\sigma} \phi\right)^{2}\right)\right)=0, \\
\mathcal{P}=-p_{a} \partial_{\sigma} a+\pi \partial_{\sigma} \phi=0,
\end{array}
$$

obey the constraint algebra similar to GR.

The action (1) could be rewritten in the form of a string on the curved background [12]

$$
S=\int d^{2} \xi \sqrt{-g} g^{\alpha \beta}(\xi) \partial_{\alpha} X^{A} \partial_{\beta} X^{B} G_{A B}(X(\xi)),
$$

where $\xi=\{\tau, \sigma\}$. The metric tensor $g_{\alpha \beta}(\xi)$ describes the intrinsic geometry of a (1+1)-dimensional manifold and $G_{A B}(X(\xi))$ describes the geometry of a background ${ }^{1}$ space. Taking $X^{A}=\{a, \phi\}$, and the metric tensors $g_{\mu v}, G_{A B}(X)$ in the form of

$$
g=\left(\begin{array}{cc}
-N^{2}+N_{1}^{2} & N_{1} \\
N_{1} & 1
\end{array}\right), \quad G=\left(\begin{array}{cc}
1 & 0 \\
0 & -a^{2}
\end{array}\right),
$$

where $N$ and $N_{1}$ are the lapse and shift functions, respectively, results in (1), which is written in the particular gauge $N=1, N_{1}=0$.

The system (4) manifests invariance relatively to the reparametrization of the variables $\tau, \sigma$ which is analog of the general transformation of coordinates in GR. Here, the $(1+1)$ - infinite string (i.e., an "immersed" space-time $\tau, \sigma)$ corresponds to the observable metric $g_{\mu v}$, while the metric $G_{A B}$ has no a physical meaning. The transformations of coordinates $\tilde{\tau}=\tilde{\tau}(\tau, \sigma), \tilde{\sigma}=\tilde{\sigma}(\tau, \sigma)$ reflects rather physical than gauge symmetry, because one living on a string could perform some experiments allowing to determine the concrete metric $g_{\mu v}$.

Considering quantization of the model (4), let us first imagine that the metric $G_{A B}$ is the Minkowski one, i.e., equals to $\operatorname{diag}\{1,-1\}$. Then, fixing the gauge by taking $g_{\mu v}$ as the Minkowski metric results in the equation of motion for $X_{\mu}$ in the form of a wave equation:

$$
X_{\mu}^{\prime \prime}-\partial_{\sigma \sigma}^{2} X_{\mu}=0
$$

Quantization of the wave equation in the Heisenberg picture allows defining the vacuum state [13]. Then one could develop a perturbation theory expanding the metric $G_{A B}$ around the Minkowski one, as it has been done for the string on the curved background [14]. Thus, by analogy with the model (4), one may suggest that the vacuum state in $\mathrm{QG}^{2}$ has to be noninvariant relatively to the general transformations of coordinates, i.e., implies some particular gauge.

1 It is not the background on which the field $\phi(\eta)$ evolves!

2 If it exists, most probably it does not exist at all. 
Let us emphasize the difference between GR and the conventional string theory ${ }^{3}$ In the string theory, the reparametrization invariance is a gauge symmetry. It does not matter for calculation of the gauge-invariant observables in which gauge they are calculated. In GR, an invariance relatively to the general transformation of coordinates has a physical meaning because, besides the gauge-invariant observables, one could also measure some quantities allowing to determine the actual system of reference (i.e., the gauge) ${ }^{4}$.

\section{Vacuum energy problem as a criterion for finding the preferred reference frame}

In the previous section, we argued that the vacuum state for the model (4) exists when the metric $g_{\mu \nu}$ is the Minkowski one. Thus, the vacuum state is not re-parametrically invariant. By analogy, one may think that the vacuum state, which is invariant relatively to the general transformation of coordinates, does not exist in QG.

The more simple problem is to define the vacuum state on the fixed background. However, even in this case, the exact vacuum state exists only for some particular space-times. In other cases, the vacuum state has only an approximate meaning $[15,16]$ and could be defined, for example, for a slowly expanding universe.

In this case, a criterion for choosing a preferred reference frame is the cosmological constant problem. This problem arises when one computes the density of vacuum energy of the quantum fields. Using the Pauli hard cutoff of the 3-momentums $k_{\max }[17,18]$ allows expanding the zero-point energy density into the different parts

$$
\begin{aligned}
& \rho_{v}=\frac{1}{4 \pi^{2} a^{4}} \int_{0}^{k_{\max }} k^{2} \sqrt{k^{2}+a^{2} m^{2}} d k \approx \\
& \frac{1}{16 \pi^{2}}\left(\frac{k_{\max }^{4}}{a^{4}}+\frac{m^{2} k_{\max }^{2}}{a^{2}}+\frac{m^{4}}{8}\left[1+2 \ln \left(\frac{m^{2} a^{2}}{4 k_{\max }^{2}}\right)\right]+\ldots\right) .
\end{aligned}
$$

The main part of this energy density $\frac{1}{16 \pi^{2}} \frac{k_{\max }^{4}}{a^{4}}$ scales as radiation. In the frame of GR, it results in an extremely fast universe expansion that contradicts the observations [19].

Mutual cancellation of this main part from bosonic and fermionic degrees of freedom demands exact supersymmetry, which does not observed to date [20]. The remanding parts in (6) are also very large, but one could suggest that there exist the sum rules for masses of bosons and fermions (the condensates should be taken into account, as well) which provide a mutual compensation of these terms $[18,21]$.

Here, we use the empirical cutoff of momentums $k_{\max }$, with a hope that some fundamental basis will be found for that in the future (like noncommutative geometry [22-24]). On the other hand, theories exist, such as the Horava-Lifshitz theory, resulting in the renormalizability of gravity. The sense of renormalization is that one changes some infinite (or very large, if $k_{\max }$ is taken finite) pieces in the operator of the mean values by some finite ones. We hope that in the future, when all the necessary particles will be discovered, the sum rules [25] will provide a mutual compensation of these infinite (or very large) pieces. That is, $k_{\max }$ will give the actual UV completions of QG without the need for renormalization.

To avoid at least the main part of the vacuum energy, one could try to build a theory of gravity in which the first term in (6) does not influence universe expansion. That could be done in some particular class of the metrics [7]

$$
d s^{2} \equiv g_{\mu \nu} d x^{\mu} d x^{\nu}=a^{2} d \eta^{2}-\gamma_{i j}\left(d x^{i}+N^{i} d \eta\right)\left(d x^{j}+N^{j} d \eta\right)
$$

3 In the conventional string theory, an observer lives in the external space-time $X_{A}$, which is 24 - or 10-dimensional before compactification [13]. The space is endowed by the metric $G_{A B}$.

4 There is a difference between the system of coordinates and the reference frame, but we will not discuss it here. 
where $x^{\mu}=\{\eta, x\}, \eta$ is a conformal time, $\gamma_{i j}$ is a spatial metric, $a=\gamma^{1 / 6}$ is a locally defined scale factor, and $\gamma=\operatorname{det} \gamma_{i j}$. As one may see, the interval (7) is of the ADM form [26], but the lapse function $N$ is equated to the determinant of the spatial metric in one-sixth degree. In the FVT [7], the Hamiltonian constraint is satisfied up to some arbitrary constant.

Eq. (7) determines the preferred reference system in which an Ether "lives." Also, one could suggest that this reference frame coincides with the reference frame in which there is no dipole anisotropy of the cosmic microwave background (CMB) [27]. Otherwise, the question arises: What is the physical foundation of a frame where $\mathrm{CMB}$ is in a rest "in tote," i.e., does not have a dipole component?
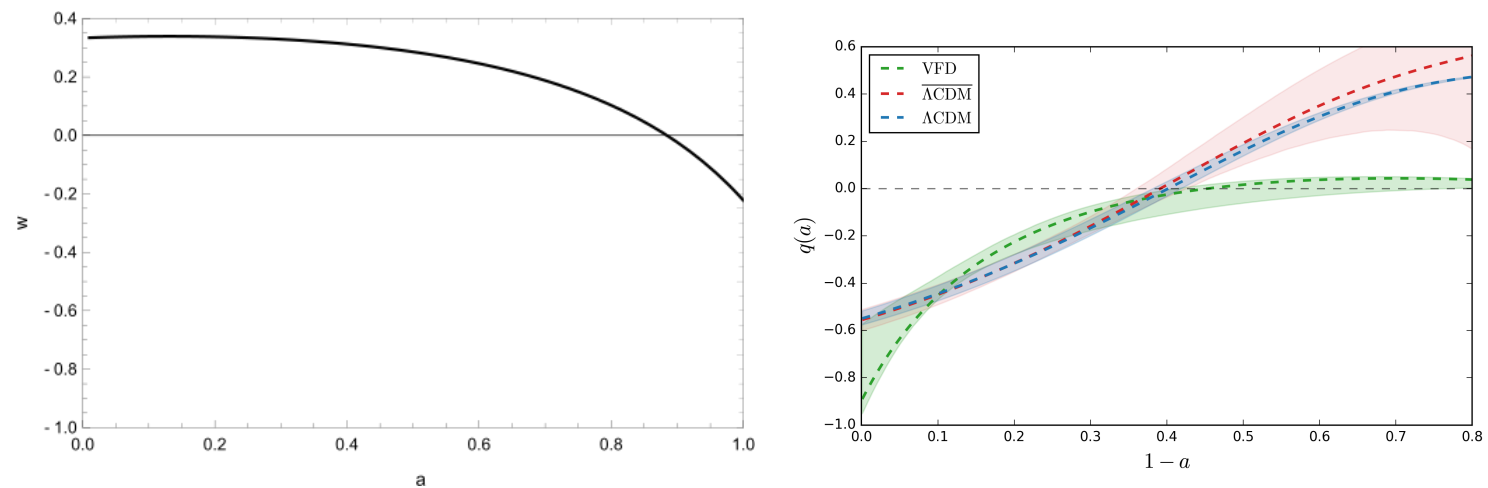

Figure 1. Left panel. Equation of the vacuum state in dependence on the universe scale factor $a$. Right panel. Deceleration parameter $q(a)$ and the corresponding dispersion channels for model (8) and two versions of $\Lambda \mathrm{CDM}$ model.

Other contributors to the vacuum energy density are the terms depending on the derivatives of the universe expansion rate [28-30]. These terms cannot be removed by any sum rules, but they have the right order of $\rho_{v} \sim M_{p}^{2} H^{2}$, where $H$ is the Hubble constant, and allow explaining the accelerated expansion of the universe. These energy density and pressure are [28-30]:

$$
\rho_{v}=\frac{a^{\prime 2}}{2 a^{6}} M_{p}^{2} S_{0}, \quad p_{v}=\frac{M_{p}^{2} S_{0}}{a^{6}}\left(\frac{1}{2} a^{\prime 2}-\frac{1}{3} a^{\prime \prime} a\right)
$$

where, $S_{0}=\frac{k_{\max }^{2}}{8 \pi^{2} M_{p}^{2}}$ is determined by the ultra-violet (UV) cut-off of the comoving momenta. The energy density and pressure of vacuum (8) satisfy a continuity equation

$$
\rho_{v}^{\prime}+3 \frac{a^{\prime}}{a}\left(\rho_{v}+p_{v}\right)=0,
$$

and, in the expanding universe, are related by the equation of state $p_{v}=w \rho_{v}$, as Fig. 1 (left panel) illustrates. Using this equation of vacuum state leads to the universe behavior at early times, when the scale factor was small, as freely rolling, i.e., without any deceleration or acceleration. That implies the zero-level deceleration parameter $q(a)$ at small scale factors $a$ [28-31], as it is shown in Fig. 1 (right panel). The discovery of an accelerated universe expansion had been a big surprise [32]. However, if the above view on a vacuum is true, one more surprise is expected. Namely, a stage preceding the acceleration should be a Milne's-like, i.e., linear in a cosmic time. The Milne's-like universes have been much discussed again recently [33-38].

\section{Size of the black hole.}

In Ref. [39], the spherically symmetric solution of the Einstein equations in the unimodular metric (7) was investigated, and it was found that the finite pressure solution exists for an arbitrary 
large mass. As a result, there are no compact objects with an event horizon ${ }^{5}$, that is, an "eicheon" appears instead of a black hole [39]. In Ref. [39], we have turned from the conformally-unimodular metric (11) to the Schwarzschild's-like metric to demonstrate that a compact object looks like a hollow sphere of the radius greater than the Schwarzschild's ones (see Fig. 2). Here, we intend to

a

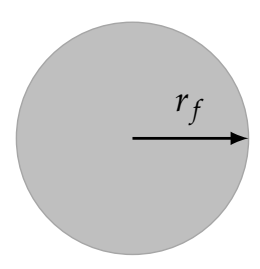

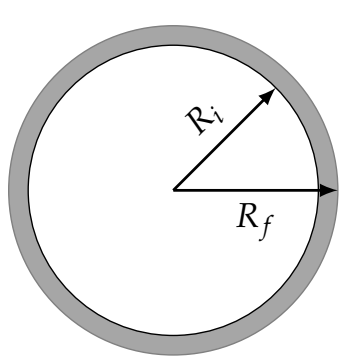

$\mathrm{b}$

Figure 2. (a) A compact object of uncompressible fluid with the radius of $r_{f}$ in the conformally-unimodular metric (11) looks as a shell (b) with the boundaries $r_{g}<R_{i}<R_{f}$ in the Schwarzschild's type metric, where $r_{g}$ is a Schwarzschild's radius.

calculate the radius of a compact object of constant density in the conformally-unimodular metric in dependence on maximum pressure and density. For this aim, it is convenient to proceed, rather, from Schwarzschild's type metric to the conformally-unimodular one. For spherically symmetric space-time, the conformally-unimodular metric (7) is reduced to

$$
d s^{2}=a^{2}\left(d \eta^{2}-\tilde{\gamma}_{i j} d x^{i} d x^{j}\right)=e^{2 \alpha}\left(d \eta^{2}-e^{-2 \lambda}(d x)^{2}-\left(e^{4 \lambda}-e^{-2 \lambda}\right)(x d x)^{2} / r^{2}\right),
$$

which looks in the spherical coordinates as

$$
d s^{2}=e^{2 \alpha}\left(d \eta^{2}-d r^{2} e^{4 \lambda}-e^{-2 \lambda} r^{2}\left(d \theta^{2}+\sin ^{2} \theta d \phi^{2}\right)\right) .
$$

Let us compare (11) with a metric of Schwarzschild's type, which has the form

$$
d s^{2}=B(R) d t^{2}-A(R) d R^{2}-R^{2}\left(d \theta^{2}+\sin ^{2} \theta d \phi^{2}\right) .
$$

The difference between the metrics (10) and (12) is that the metric (12) suggests that the circumference equals $2 \pi R$. However, there is no experimental evidence for this fact in the arbitrary spherically symmetric space-time. For the metric (10) the circumference is not equal to $2 \pi r$ in the close vicinity of the point-like mass, but this metric solves a part of the vacuum energy problem. Coordinate transformation $t=\eta, R=R(r)$ relates the metrics (11) and (12):

$$
\begin{gathered}
B(R)=1-r_{g} / R=e^{2 \alpha} \\
R^{2}=r^{2} e^{-2 \lambda+2 \alpha} \\
A(R)\left(\frac{d R}{d r}\right)^{2}=e^{4 \lambda+2 \alpha} .
\end{gathered}
$$

Using (13), (14) in (15) to exclude $\lambda$ and $\alpha$ yields

$$
\frac{d r}{d R}=\frac{R^{2}}{r^{2}} \frac{A^{1 / 2}}{B^{3 / 2}} .
$$

\footnotetext{
5 The event horizon is a region of space-time which is causality disjointed from the rest of space-time.
} 
For an empty Schwarzschild space-time $A(R)=\left(1-r_{g} / R\right)^{-1}$ and $B(R)=1-r_{g} / R$, while, in the region filled by matter, $A(R)$ and $B(R)$ obey [40]

$$
\begin{aligned}
\frac{d}{d R}\left(\frac{R}{A}\right) & =1-\frac{6}{M_{p}^{2}} \rho R^{2}, \\
\frac{1}{B} \frac{d B}{d R} & =-\frac{2}{p+\rho} \frac{d p}{d R},
\end{aligned}
$$

where the reduced Planck mass $M_{p}=\sqrt{\frac{3}{4 \pi G}}$ and $r_{g}=\frac{3 m}{2 \pi M_{p}^{2}}$. Further, as in [39], we will consider a model of the constant density $\rho(R)=\rho_{0}$. In this case Eqs. (17) and (18) can be integrated explicitly that gives

$$
\begin{aligned}
A & =\frac{R}{R-1-2 \rho_{0}\left(R^{3}-R_{f}^{3}\right)} \\
B & =\left(1-\frac{1}{R_{f}}\right) \frac{\rho_{0}^{2}}{\left(p(R)+\rho_{0}\right)^{2}}
\end{aligned}
$$

and one needs only to find a pressure, which obeys the Tolman-Volkov-Oppenheimer (TVO) equation

$$
p^{\prime}(R)=-\frac{3}{4 \pi M_{p}^{2} R^{2}} \mathcal{M}(R) \rho(R)\left(\frac{1+4 \pi R^{3} p(R)}{\mathcal{M}(R)}\right)\left(1+\frac{p(R)}{\rho(R)}\right)\left(1-\frac{3 \mathcal{M}(R)}{2 \pi M_{p}^{2} R}\right)^{-1} .
$$

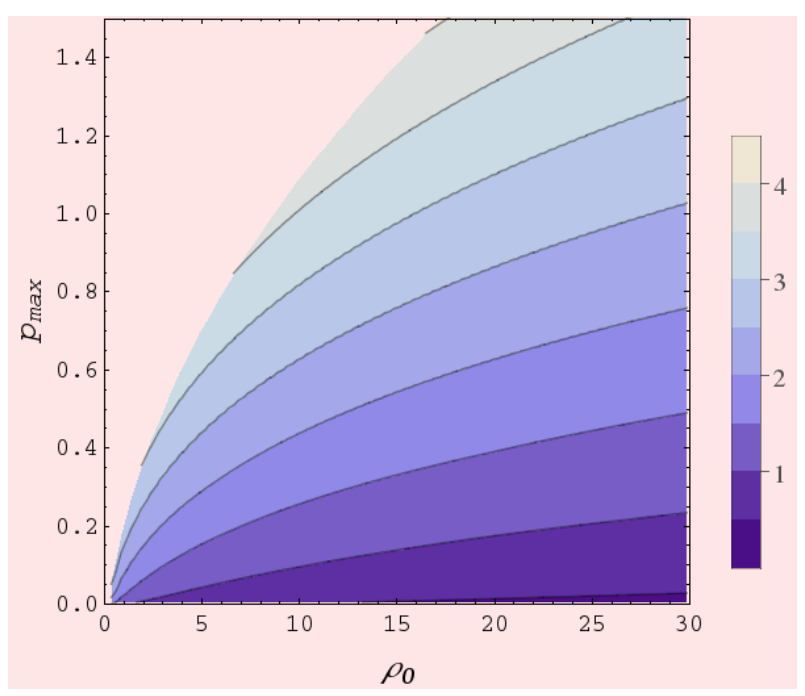

Figure 3. The eicheon radius $r_{f}=r\left(R_{f}\right)$, expressed in the units of gravitational radius, in the conformally unimodular metric in dependence on density and maximal pressure (i.e., pressure in the center of eicheon). Pressure and density are in the units of $M_{p}^{2} r_{g}^{-2}$.

Here, we measure density and pressure in the units of $M_{p}^{2} r_{g}^{-2}$, which is convenient, because the mean density of Schwarzschild black hole equals $1 / 2$, while the Tolmen-Oppenheimer-Volkov (TOV) limit $R_{f}<\frac{9}{8} r_{g}$ gives the value of $\rho_{0}=\frac{1}{2}\left(\frac{8}{9}\right)^{3} \approx 0.35$. Using

$$
\mathcal{M}(R)=\frac{4 \pi}{3} \rho_{0}\left(R^{3}-R_{i}^{3}\right),
$$

for solving the TOV equation for pressure, it is possible to find $B$, and then solve (16) with the initial condition $r\left(R_{i}\right)=0$ to find the eicheon radius $r_{f}=r\left(R_{f}\right)$ in the conformally unimodular metric. As for 
the eicheon radius in Schwarzschild's type metric, it is equal $R_{f}=\sqrt[3]{R_{i}^{3}+\frac{1}{2 \rho_{0}}}$ in the units of $r_{g}$, where $R_{i}$ is an inner radius, which determines maximum pressure. The closer $R_{i}$ to unity the greater maximal pressure. Let us to plot (see Fig. 3) calculated radius of the eicheon in the conformally unimodular metric in dependence of density $\rho_{0}$ and maximum pressure, that is the pressure in the center of a solid ball in the metric (11) .

\section{Decoherence of the particles due to gravitational potential fluctuations.}
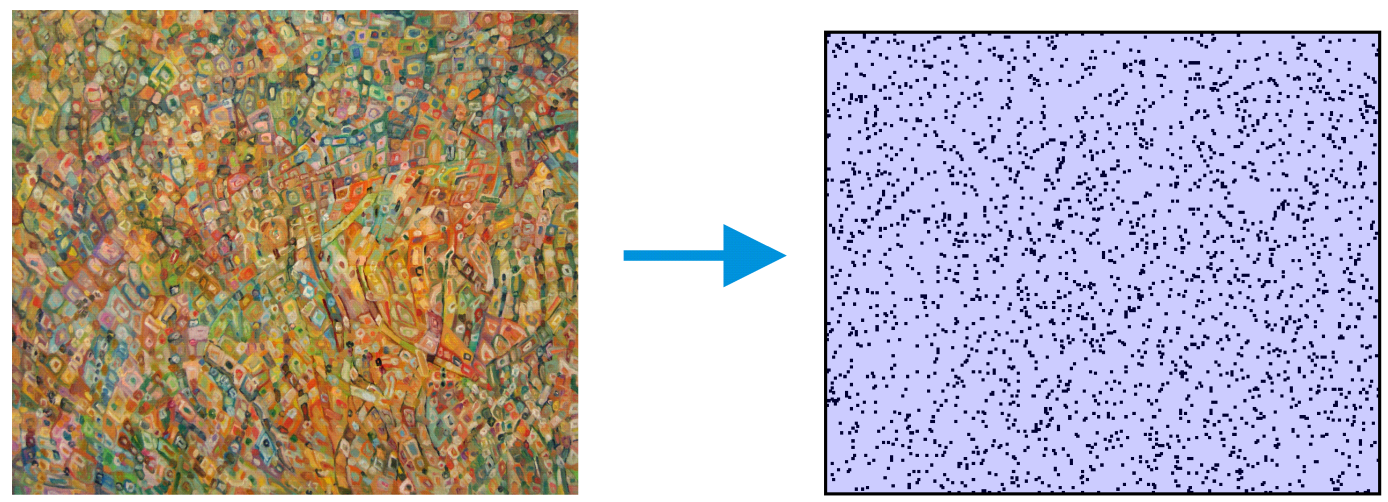

Figure 4. Under the Minkowski's background, the QG is reducible to a number of randomly positioned delta-like sources of the gravitational field (abstract artwork by Oksana Cherkas is used at the left panel).

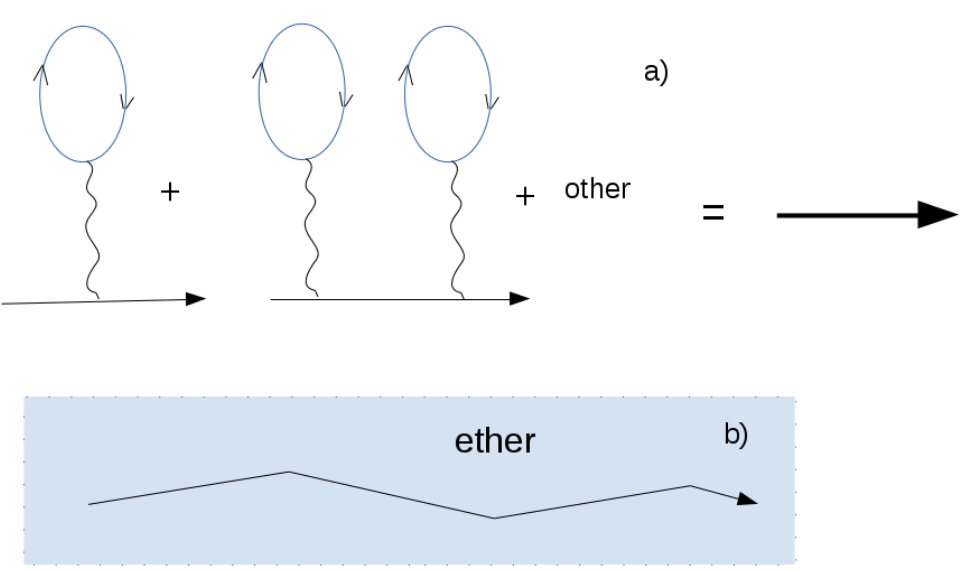

Figure 5. Illustration of vacuum influence on the particle propagation in a) quantum field theory, where the vacuum loops renormalize mass and charge of the particle but do not prevent them freely motion, and b)QG, where space is filled by ether due to the absence of a vacuum state.

One more implication of the conformally-unimodular metric arises for the table-top quantum gravity experiments [41]. In GR, it would not be possible to say that vacuum fluctuations under Minkowski's space-time are small. Actually, for the small vacuum fluctuations, one could turn to the reference system where they are significant. That means the appearance of so-called gauge waves, which are the consequence of the reference frame choice. By restricting the possible reference systems, it would be possible to see the actual vacuum fluctuation influencing the motion of the massive particles. 
Under Minkowski's background, one could write

$$
a(\eta, r)=(1+\Phi(\eta, r))
$$

As was shown [41], the operator of the gravitational potential in a vacuum has the correlator

$$
<0\left|\hat{\Phi}(\boldsymbol{r}, \eta) \hat{\Phi}\left(\boldsymbol{r}^{\prime}, \eta\right)\right| 0>\sim \delta\left(\boldsymbol{r}-\boldsymbol{r}^{\prime}\right) / M_{p}^{3}
$$

That means, as is shown schematically in Fig. 4, that fluctuations are similar to a random medium consisting of the sources of a gravitational potential with contact interaction which influences a massive moving particle. As a result, nonrelativistic massive particle waves lose their coherence. However, more careful consideration will be needed because a correlator taken at different times $<0\left|\hat{\Phi}(r, \eta) \hat{\Phi}\left(r^{\prime}, \eta^{\prime}\right)\right| 0>$ should figurative in the decoherence theory.

The difference of particle propagation in QG and QFT is illustrated in Fig. 5. Again we emphasize that the ether in QG originates from an absence of the vacuum state invariant relatively to the general transformation of coordinates, and, in particular, relatively to the Lorentz transformation, when they are considered as a subgroup of the general transformation of coordinates.

\section{Conclusion}

To summarize, the conformally unimodular metric gives the sustained basis for considering both quantum gravity physics and compact astrophysical objects. Although such a fascinating physics as closed time-like curves [42-44], time machines [45,46], wormholes [47], Hawking radiation by the horizon [48] is excluded in the conformally unimodular metric, this metric gives fresh impetus to investigation real physical phenomena, including structure formation [30], CMB physics [49], investigation of the structure of eicheons, search for the decoherence QG effects and other QG consequences of the vacuum fluctuations of the gravitational potential, implying that this singled out metric corresponds to a system of reference, where the ether is at rest "in tote." Certainly, it suggests the ether existence per se.

1. Schaffner, K.F. Nineteenth-Century Aether Theories; Pergamon: NY, 1972.

2. Berestetskii, V.B.; Landau, L.; Lifshitz, E.; Pitaevskii, L.P. Quantum Electrodynamics; Butterworth-Heinemann: Oxford, 1982.

3. Jacobson, T. Einstein-Aether gravity: a status report. Proc. Sci. 2008, QG-Ph, 020. doi:10.22323/1.043.0020.

4. Horava, P. Quantum gravity at a Lifshitz point. Phys. Rev. D 2009, 79. doi:10.1103/physrevd.79.084008.

5. Nilsson, N.A.; Czuchry, E. Horava-Lifshitz cosmology in light of new data. Phys. Dark Univ. 2019, 23, 100253. doi:10.1016/j.dark.2018.100253.

6. Nilsson, N.A. Aspects of Lorentz and CPT Violation in Cosmology. PhD thesis, National Centre for Nuclear Research, Otwock-Świerk, 2020.

7. Cherkas, S.L.; Kalashnikov, V.L. An approach to the theory of gravity with an arbitrary reference level of energy density. Proc. Natl. Acad. Sci. Belarus, Ser. Phys.-Math. 2019, 55, 83-96, [arXiv:gr-qc/1609.00811].

8. Tureanu, A. CPT and Lorentz Invariance: Their Relation and Violation. J. Phys.: Conf. Ser. 2013, 474, 012031. doi:10.1088/1742-6596/474/1/012031.

9. Cherkas, S.L.; Batrakov, K.G.; Matsukevich, D. Testing of CP, CPT, and causality violation with light propagation in vacuum in the presence of uniform electric and magnetic fields. Phys. Rev. D 2002, 66. doi:10.1103/physrevd.66.065011.

10. Kostelecky, V.A.; Russell, N. Data tables for Lorentz and CPT violation. Rev. Mod. Phys. 2011, 83, 11-31. doi:10.1103/revmodphys.83.11.

11. Kostelecky, V.A.; Mewes, M. Lorentz and diffeomorphism violations in linearized gravity. Phys. Lett. B 2018, 779, 136-142. doi:10.1016/j.physletb.2018.01.082. 
12. Cherkas, S.L.; Kalashnikov, V.L. An inhomogeneous toy model of the quantum gravity with the explicitly evolvable observables. Gen. Rel. Grav. 2012, 44, 3081-3102. doi:10.1007/s10714-012-1441-5.

13. Kaku, M. Introduction to Superstrings; Springer: New York, 2012.

14. Green, M.B.; Schwarz, J.; Witten, E. Superstring Theory; Vol. 1, Univ. Press: Cambridge, 1987.

15. Birrell, N.D.; Davies, P.C.W. Quantum Fields in Curved Space; Cambridge Monographs on Mathematical Physics, Cambridge University Press, 1982. doi:10.1017/CBO9780511622632.

16. Anischenko, S.; Cherkas, S.; Kalashnikov, V. Functional minimization method addressed to the vacuum finding for an arbitrary driven quantum oscillator. Nonlin. Phenom. Compl. Syst. 2009, 12, 16-26, [arXiv:quant-ph/0806.1593].

17. Pauli, W. Pauli Lectures on Physics: Vol 6, Selected Topics in Field Quantization; MIT Press: Cambridge, 1971.

18. Visser, M. Lorentz Invariance and the Zero-Point Stress-Energy Tensor. Particles 2018, 1, 138-154, [arXiv:gr-qc/1610.07264]. doi:10.3390/particles1010010.

19. Blinnikov, S.I.; Dolgov, A.D. Cosmological acceleration. Phys. Usp. 2019, 62, 529. doi:10.3367/ufne.2018.10.038469.

20. Autermann, C. Experimental status of supersymmetry after the LHC Run-I. Progr.Part. Nucl. Phys. 2016, 90, 125-155. doi:10.1016/j.ppnp.2016.06.001.

21. Cherkas, S.; Kalashnikov, V. The equation of vacuum state and the structure formation in universe. Nonlin. Phenom. Complex Syst. 2020, 23, 332-337, [arXiv:gr-qc/1810.06211].

22. Kowalski-Glikman, J.; Nowak, S. Non-commutative space-time of Doubly Special Relativity theories. Int. J. Mod. Phys. D 2003, 12, 299-315. doi:10.1142/s0218271803003050.

23. Pachol, A. Short review on noncommutative spacetimes. J. Phys.: Conf. Ser. 2013, 442, 012039. doi:10.1088/1742-6596/442/1/012039.

24. Mir-Kasimov, R.M. Noncommutative space-time and relativistic dynamics. Phys. Part. Nucl. 2017, 48, 309-318. doi:10.1134/S1063779617020058.

25. Visser, M. The Pauli sum rules imply BSM physics. Physics Letters B 2019, 791, $43-47$. doi:10.1016/j.physletb.2019.02.010.

26. Arnowitt, R.; Deser, S.; Misner, C.W. Republication of: The dynamics of general relativity. Gen. Rel. Grav. 2008, 40, 1997-2027. doi:10.1007/s10714-008-0661-1.

27. Dodelson, S. Modern Cosmology; Elsevier: Amsterdam, 2003.

28. Cherkas, S.L.; Kalashnikov, V.L. Determination of the UV cut-off from the observed value of the Universe acceleration. JCAP 2007, 01, 028, [arXiv:gr-qc/gr-qc/0610148]. doi:10.1088/1475-7516/2007/01/028.

29. Cherkas, S.L.; Kalashnikov, V.L. Universe driven by the vacuum of scalar field: VFD model. Proc. Int. conf. "Problems of Practical Cosmology",Saint Petersburg, Russia, June 23 - 27, 2008, 2008, pp. 135-140, [arXiv:astro-ph/astro-ph/0611795].

30. Cherkas, S.L.; Kalashnikov, V.L. The equation of vacuum state and the structure formation in universe. Nonlin. Phenom. Compl. Syst. 2020, 23, 332-337, [arXiv:gr-qc/1810.06211].

31. Haridasu, B.S.; Cherkas, S.L.; Kalashnikov, V.L. A reference level of the Universe vacuum energy density and the astrophysical data. Fortschr. Phys. 2020, 68, 2000047, [1912.09224]. doi:10.1002/prop.202000047.

32. Perlmutter, S. Supernovae, Dark Energy, and the Accelerating Universe. Physics Today 2003, 56, 53. doi:10.1063/1.1580050.

33. Sultana, J. The Rh = ct universe and quintessence. MNRAS 2016, 457, 212-216. doi:10.1093/mnras/stv3012.

34. Klinkhamer, F.R.; Wang, Z.L. Instability of the big bang coordinate singularity in a Milne-like universe, 2019, [arXiv:gr-qc/1911.11116].

35. Wan, H.Y.; Cao, S.L.; Melia, F.; Zhang, T.J. Testing the Rh=ct universe jointly with the redshift-dependent expansion rate and angular-diameter and luminosity distances. Phys. Dark Univ. 2019, 26, 100405. doi:10.1016/j.dark.2019.100405.

36. Manfredi, G.; Rouet, J.L.; Miller, B.N.; Chardin, G. Structure formation in a Dirac-Milne universe: Comparison with the standard cosmological model. Physical Review D 2020, 102. doi:10.1103/physrevd.102.103518.

37. Lewis, G.F.; Barnes, L.A. The one-way speed of light and the Milne universe. Publications of the Astronomical Society of Australia 2021, 38, e007. doi:10.1017/pasa.2021.2. 
38. Chardin, G.; Dubois, Y.; Manfredi, G.; Miller, B.; Stahl, C. MOND-like behavior in the Dirac-Milne universe - Flat rotation curves and mass/velocity relations in galaxies and clusters, 2021, [arXiv:astro-ph.GA/2102.08834].

39. Cherkas, S.L.; Kalashnikov, V.L. Eicheons instead of Black holes. Phys. Scr. 2020, 95, 085009. doi:10.1088/1402-4896/aba3aa.

40. Weinberg, S. Gravitation and Cosmology: Principles and Applications of the General Theory of Relativity; John Wiley \& Sons: New York, 1972.

41. Cherkas, S.L.; Kalashnikov, V.L. Wave optics of quantum gravity for light and particles, 2021, [arXiv:gr-qc/2012.02288].

42. Gonzalez-Diaz, P.; Garay, L. Quantum Closed Timelike Curves in General Relativity. In Recent Developments in General Relativity; Cianci, R.; Collina, R.; Francaviglia, M.; Fre, P., Eds.; Springer: Genoa, 2002; pp. 459-463. doi:10.1007/978-88-470-2101-3_41.

43. Bini, D.; Geralico, A. On the occurrence of Closed Timelike Curves and the observer's point of view. European Physical Journal Web of Conferences, 2013, Vol. 58, European Physical Journal Web of Conferences, p. 01002. doi:10.1051/epjconf/20135801002.

44. Faizuddin, A. Type III spacetime with closed timelike curves. Progr. Phys. 2016, 12, 329-331.

45. Novikov, I. An analysis of the operation of a time machine. JETP 1989, 95, 439-443.

46. Wuthrich, C. Time travelling in emergent spacetime, 2019, [arXiv:physics.hist-ph/1907.11167].

47. Teo, E. Rotating traversable wormholes. Phys. Rev. D 1998, 58. doi:10.1103/physrevd.58.024014.

48. Coogan, A.; Morrison, L.; Profumo, S. Direct Detection of Hawking Radiation from Asteroid-Mass Primordial Black Holes. Phys. Rev. Lett. 2021, 126. doi:10.1103/physrevlett.126.171101.

49. Cherkas, S.L.; Kalashnikov, V.L. Plasma perturbations and cosmic microwave background anisotropy in the linearly expanding Milne-like universe. In Fractional Dynamics, Anomalous Transport and Plasma Science; Skiadas, C.H., Ed.; Springer: Cham, 2018; chapter 9. doi:10.1007/978-3-030-04483-1_9. 